\title{
Numerical simulation of intergranular and transgranular crack propagation in ferroelectric polycrystals
}

\author{
Amir Abdollahi · Irene Arias
}

Received: 15 September 2011 / Accepted: 29 November 2011 / Published online: 17 January 2012

(C) Springer Science+Business Media B.V. 2012

\begin{abstract}
We present a phase-field model to simulate intergranular and transgranular crack propagation in ferroelectric polycrystals. The proposed model couples three phase-fields describing (1) the polycrystalline structure, (2) the location of the cracks, and (3) the ferroelectric domain microstructure. Different polycrystalline microstructures are obtained from computer simulations of grain growth. Then, a phase-field model for fracture in ferroelectric single-crystals is extended to polycrystals by incorporating the differential fracture toughness of the bulk and the grain boundaries, and the different crystal orientations of the grains. Our simulation results show intergranular crack propagation in fine-grain microstructures, while transgranular crack propagation is observed in coarse grains. Crack deflection is shown as the main toughening mechanism in the fine-grain structure. Due to the ferroelectric domain switching mechanism, noticeable fracture toughness enhancement is also obtained for transgranular crack propagation. These observations agree with experiment.
\end{abstract}

Keywords Polycrystals · Ferroelectricity · Fracture Phase-field models · Finite element analysis

A. Abdollahi · I. Arias $(\varangle)$

Laboratori de Càlcul Numèric (LaCàN), Departament de

Matemàtica Aplicada III, Universitat Politècnica de

Catalunya (UPC), Campus Nord UPC-C2,

08034 Barcelona, Spain

e-mail: irene.arias@upc.edu

\section{Introduction}

The design and implementation of electromechanical systems demand multifunctional materials with strong electromechanical coupling and reasonable reliability. In this category ferroelectric ceramics are the main candidates also exhibiting short response times. However, due to their inherent brittleness, a deep understanding of the fracture behavior of these materials is key to assure optimum reliability of the systems. Towards this goal, numerous theoretical and experimental investigations have been carried out during the past decades. Excellent reviews of these works are presented in (Zhang and Gao 2004; Schneider 2007; Kuna 2010). Ferroelectric ceramics exhibit ferroelectric and ferroelastic switching behavior with macroscopic dielectric and butterfly hystereses due to the underlying microscopic mechanisms. The reviews in (Kamlah 2001; Huber 2005) present related modeling approaches. The formation and evolution of the microstructure are more probable under high electromechanical loadings and near load concentrations such as the vicinity of cracks (Hackemann and Pfeiffer 2003; Jones et al. 2007). Interactions between the microstructure, grain boundaries, localized stress and electric fields near the crack tips lead to the complexity of fracture phenomena in ferroelectric polycrystals. Several approaches have been proposed to study the fracture in ferroelectric materials, including phenomenological constitutive models (Landis 2003; Sheng and Landis 2007; Wang and Landis 2006), models investigating 
the local phase transformations near the crack tip (Zhu and Yang 1997, 1999; Yang and Zhu 1998), phasefield models for computing the mechanical and electromechanical $J$-integrals (Song et al. 2007; Wang and Zhang 2007, 2008; Li and Landis 2011; Li and Kuna 2011), and more recently, a phase-field model accurately accounting for the stray fields (Yang and Dayal 2011). See also (DeSimone et al. 2001) for a related approach in ferromagnetics. However, these models assume fixed or stationary crack configurations and they are unable to capture the interactions between a propagating crack and the microstructure of the material. To tackle the full complexity of the phenomenon, we have recently introduced a phase-field model for the coupled microstructure and fracture evolution in ferroelectric single crystals (Abdollahi and Arias 2011b). We have shown that the interaction of the microstructure and the crack leads to a slow-fast crack propagation behavior observed in experiments. In another work, we have presented evidence that this interaction results in the anisotropy of the fracture toughness (Abdollahi and Arias 2011c). A related phase-field approach has
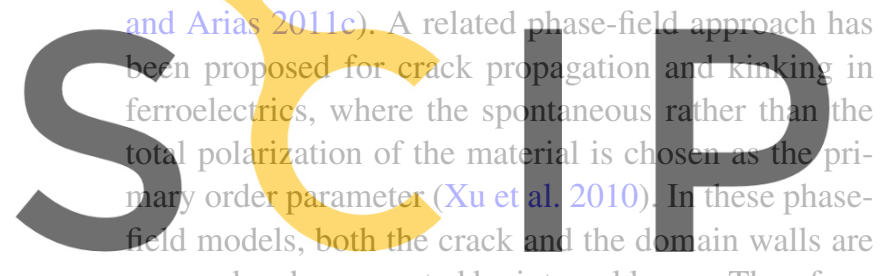

smeared and represented by internal layers. Therefore,

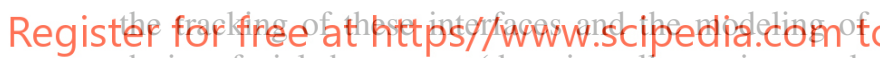
the interfacial phenomena (domain wall energies, crack face boundary conditions) are automatically accomplished by the governing equations. Nevertheless, this flexibility comes at the expense of a high computational cost, since the width of the phase-field regularizations of the domain wall and the crack must be resolved by the discretization.

To the best of our knowledge, no model has been proposed in the literature to study the interactions between the fracture process and the polycrystalline and domain structure in ferroelectric polycrystals. The related models (Landis 2003; Li and Kuna 2011) have only taken into account the grains orientations, but the effects of grain boundaries and grain size have not been considered. In (Verhoosel and Gutierrez 2009), the grain microstructure is introduced to model inter- and transgranular crack propagation in linear piezoelectric polycrystals, thus not accounting for the effect of ferroelectric domain microstructures. The main objective of this paper is to study the fracture processes in ferroelec- tric polycrystals in its full complexity, and in particular to evaluate the effects produced by the grain and ferrolelectric domain microstructures on the fracture response of the material. For this purpose, we extend to polycrystals the phase-field model of fracture in ferroelectric single crystals that we have recently proposed (Abdollahi and Arias 2011a, b, c). The results obtained with this model prove its potential to capture complex interactions between the crack and the material microstructure. The theory of the model for polycrystals is summarized in Sect. 2. Numerical simulations are presented in Sect. 3, along with a qualitative discussion of the observed fracture mechanisms and their relation to the polycrystalline and the ferroelectric domain microstructures. The last section is the conclusion of this paper.

\section{Theory}

The proposed model for fracture of ferroelectric polycrystals is based on two existing phase-field approaches
for: (1) grain growth (Fan and Chen 1997) and (2) frac-
ture in ferroeldetric singlecrystals (Abdollahi ad Arias
$2011 \mathrm{~b}$ ). The first model provides realistic polycystal-
line microstructures withdifferent grain size while the
second models the complex interactions between the

fracture processes and the ferroelectric domain forma-

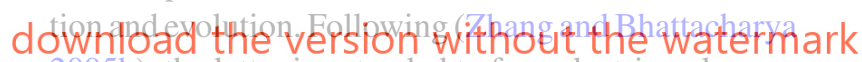
2005b), the latter is extended to ferroelectric polycrystals. The resulting model couples three phase-fields describing (1) the polycrystalline structure, (2) the location of the cracks, and (3) the ferroelectric domain microstructure. In this section, the phase-field models for grain growth and fracture of ferroelectric single crystals are briefly discussed. Then, the extension to ferroelectric polycrystals and its numerical implementation are described.

\subsection{Phase-field model for grain growth}

According to the phase-field model of grain growth (Fan and Chen 1997), the total free energy of a heterogeneous system is stated as

$$
F=\int_{\Omega}\left[f_{0}\left(\eta_{1}, \eta_{2}, \ldots, \eta_{m}\right)+\sum_{i=1}^{m} \frac{\kappa_{i}}{2}\left(\nabla \eta_{i}\right)^{2}\right] \mathrm{d} \Omega,
$$


where $f_{0}$ is the local free energy density associated with the orientation field variables $\left(\eta_{1}, \eta_{2}, \ldots, \eta_{m}\right)$ for distinguishing different orientations of grains and $m$ is the number of possible orientations. $\kappa_{i}$ are the coefficients of the gradient energy terms penalizing sharp variations in the field variables. The free energy density $f_{0}$ is written as (Fan and Chen 1997)

$$
\begin{aligned}
f_{0}\left(\eta_{1}, \eta_{2}, \ldots, \eta_{m}\right)= & \sum_{i=1}^{m}\left(-\frac{\alpha}{2} \eta_{i}^{2}+\frac{\beta}{4} \eta_{i}^{4}\right) \\
& +\gamma \sum_{i=1}^{m} \sum_{j \neq i}^{m} \eta_{i}^{2} \eta_{j}^{2},
\end{aligned}
$$

where $\alpha, \beta$ and $\gamma$ are positive constants characterizing the energy landscape. For $\gamma>\beta / 2, f_{0}$ furnishes a multi-well energy landscape with $2 m$ minima located at $\left(\eta_{1}, \eta_{2}, \ldots, \eta_{m}\right)=(1,0, \ldots, 0),(0,1, \ldots, 0), \ldots$, $(0,0, \ldots, 1),(-1,0, \ldots, 0),(0,-1, \ldots, 0), \ldots,(0$, $0, \ldots,-1)$. Each of these $2 m$ minima corresponds to a number of grains constructing a part of the polycrystalline microstructure. The grain boundaries are the regions of the domain where the gradient energy terms are non-zero. The evolution of the grains is governed by the time-dependent Ginzburg-Landau (TDGL) equa-
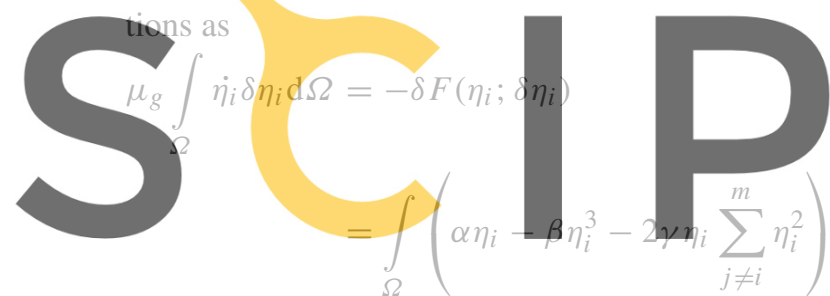

$\mathrm{d} \Omega$

Register for free at http\$//www.scipedia.com to $+\int_{\Omega}\left(\kappa_{i} \nabla^{2} \eta_{i}\right) \mathrm{d} \Omega$

where $i=1,2, \ldots, m$ and $1 / \mu_{g}$ is the mobility of the process.

\subsection{Phase-field model for fracture of ferroelectric single crystals}

In the spirit of Francfort and Marigo (1998) and Shu and Bhattacharya (2001), the total electromechanical enthalpy of a possibly fractured ferroelectric single crystal occupying a region $\Omega$ is stated as (Abdollahi and Arias 2011b)

$$
\begin{aligned}
H[\mathbf{u}, v, \mathbf{p}, \phi]= & \int_{\Omega} h(\boldsymbol{\varepsilon}(\mathbf{u}), \mathbf{p}, \nabla \mathbf{p}, \mathbf{E}(\phi), v) \mathrm{d} \Omega \\
& +G_{c} \int_{\Omega}\left[\frac{(1-v)^{2}}{4 \kappa}+\kappa|\nabla v|^{2}\right] \mathrm{d} \Omega,
\end{aligned}
$$

where body loads, volume charges, tractions and surface charges have been ignored for simplicity. The first integral is referred to as total bulk energy of the material while the second integral takes the role of the surface energy. The constant $G_{c}$ is the critical energy release rate or the surface energy density in Griffith's theory (Griffith 1921). The scalar field $v$ is the phasefield parameter describing a smooth transition in space between unbroken $(v=1)$ and broken $(v=0)$ states of the material. When the positive regularization parameter $\kappa$ tends to zero, this transition becomes sharper. The electro-mechanical enthalpy density $h$ is given as

$$
\begin{aligned}
h(\boldsymbol{\varepsilon}, \mathbf{p}, \nabla \mathbf{p}, \mathbf{E}, v)= & \left(v^{2}+\eta_{\kappa}\right)[U(\nabla \mathbf{p})+W(\mathbf{p}, \boldsymbol{\varepsilon})] \\
& +\chi(\mathbf{p})-\frac{\varepsilon_{0}}{2}|\mathbf{E}|^{2}-\mathbf{E} \cdot \mathbf{p},
\end{aligned}
$$

where $\varepsilon$ is the strain tensor associated with the mechanical displacement $\mathbf{u}, \varepsilon=1 / 2\left(\nabla \mathbf{u}+\nabla^{T} \mathbf{u}\right), \mathbf{p}$ is the polarization, $\mathbf{E}$ is the electric field associated with the electric potential $\phi$ as $\mathbf{E}=-\nabla \phi$ and $\varepsilon_{0}$ is the permittivity of free space. $\eta_{\kappa}$ is a small (relative to $\kappa$ ) residual stiffness to avoid the singularity of the first part of the energy in fully fractured regions of the domain. $U$ is the

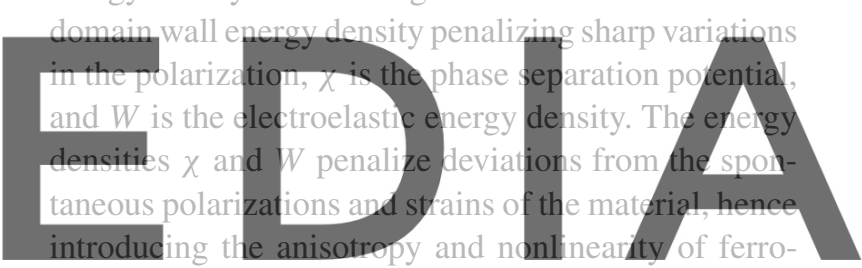

electric materials.

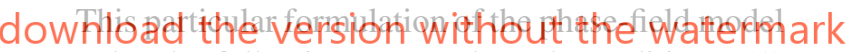

encodes the following assumed crack conditions: (1) traction free, (2) electrically permeable, and (3) free polarization (Abdollahi and Arias 2011a). For a sharp crack, the traction-free conditions are stated as $\sigma \cdot \mathbf{n}=\mathbf{0}$ where $\sigma$ and $\mathbf{n}$ are the stress tensor and the unit outward normal on the crack faces, respectively. The permeable crack conditions assume that the crack does not perturb the electrical fields and the electric potential and the normal component of the electric displacement are continuous across the crack faces, i.e.

$\phi^{+}=\phi^{-} \quad$ and $\quad \mathbf{D}^{+} \cdot \mathbf{n}^{+}=\mathbf{D}^{-} \cdot \mathbf{n}^{-}$,

where the superscripts + and - denote the top and bottom crack faces. The free-polarization boundary conditions imply that the gradients normal to the crack faces of the polarization components vanish at the crack faces, i.e.

$\frac{d p_{i}^{+}}{d n}=\frac{d p_{i}^{-}}{d n}=0,(i=1,2)$. 
It can be shown numerically that all of these conditions are satisfied in the vicinity of the smeared crack for a finite but small value of the regularization parameter $\kappa$, as expected in the sharp crack model (Abdollahi and Arias 2011b). Other electro-mechanical crack conditions can be modeled similarly (Abdollahi and Arias 2011a).

The energy terms are chosen following (Devonshire 1949 , 1951) for the case of plane polarization and plane strain as
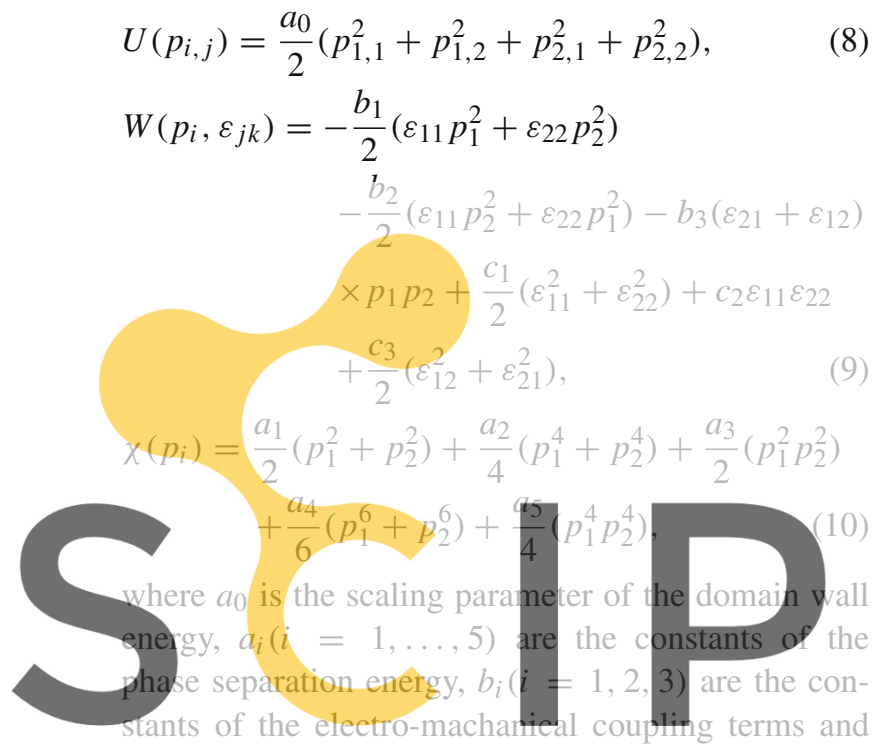

$c_{i}(i=1,2,3)$ are the elastic constants. The combi-

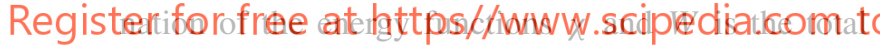
Landau-Devonshire energy density furnishing a multiwell energy landscape with four minima corresponding to the four variants of the tetragonal phase. The time evolution of the governing equations along with a simple algorithm to solve the coupled system are presented in Sect. 2.3, for the polycrystal model.

\subsection{Phase-field model for fracture of ferroelectric polycrystals}

The total electromechanical enthalpy in Eq. (4) is extended here to study the crack propagation in ferroelectric polycrystals. For this purpose, the polycrystalline microstructure given by the model of grain growth described in Sect. 2.1 is included in the formulation as follows. In the models of brittle polycrystalline materials, the fracture properties of the grain boundaries can be characterized by assuming a lower fracture toughness along these boundaries as compared to the grain interiors. Other works (Grah et al. 1996; Sukumar et al. 2003; Verhoosel and Gutierrez 2009) have proven the validity of this assumption for the study of the intergranular and the transgranular modes of fracture. In the model of grain growth described in Sect. 2.1, the grain boundaries can be identified through a function $\xi$ of the orientation field variables $\eta_{i}$ defined as

$\xi=\sum_{i=1}^{m} \eta_{i}^{2 k}$,

where $k$ is a positive integer. The function $\xi$ with $k=1$ is commonly used to visualize the polycrystalline microstructures. It has a unit value inside the grains and smaller positive values at the grain boundaries (Fan and Chen 1997). Through this function, we can easily assign a lower fracture toughness along the grain boundaries than in the grain interiors. The critical energy release rate in a polycrystal can be defined as

$G_{c}^{\text {poly }}=G_{c} \mathscr{F}(\xi)$,

where $G_{C}$ is the critical energy release rate of the bulk crystal, and the function $F$ controls the wealening
of the material at the grain boundaries. This fanction
attains its maximum for $\xi=1$ (inside the grains) and
its minimum indicates the ratio of the critical fracture
energy of the grain boundary to that of the grain interior. By changing this ratio, it is possible to study the transition between the intergranular and transgranular modes downlagd the versign without the watermark

et al. 2003). This ratio can be regulated in our model by an appropriate selection of the function $\mathscr{F}$ and setting the power $k$ in Eq. (11) and the coefficients of the gradient energy $\left(\kappa_{i}\right)$. Here, the function $\mathscr{F}$ is chosen to be proportional to $\xi$, i.e. $\mathscr{F}(\xi)=\xi$. The power of $\xi$ is also assigned as $k=1$ to avoid decreasing the fracture toughness towards zero along the grain boundaries, which is unphysical. Note that other modeling options are possible, but in the absence of precise information about the grain boundary properties, the choices of the function $\mathscr{F}$ and power $k$ seem adequate.

We also assume that each grain is oriented along a different crystallographic direction. In $2 \mathrm{D}$, the rotation angle $\Theta$ is defined and its value is assigned randomly for each orientation field variables $\eta_{i}(i=1, \ldots, m)$. Then, the rotation field over a polycrystalline microstructure can be obtained as

$\theta=\frac{\sum_{i=1}^{m} \Theta_{i} \eta_{i}^{2}}{\sum_{i=1}^{m} \eta_{i}^{2}}$. 
The function $\theta$ has the value $\Theta_{i}$ inside the grains corresponding to the orientation field variable $\eta_{i}$. At the grain boundaries, the numerator in Eq. (13) decreases significantly. The function $\xi$ in the denominator compensates for this effect and assures a smooth transition between the orientations of adjacent grains across their boundaries.

Using the rotation field $\theta$ and following the phasefield model of ferroelectric polycrystals (Zhang and Bhattacharya 2005b), the electromechanical enthalpy density $h$ in Eq. (5) can be extended to polycrystals. For this purpose, each of the energy terms in Eq. (5) is transformed from the local coordinate system of each individual grain to the global coordinate system of the polycrystal, $\theta$ being the angle between these two coordinate systems. It ean be shown that the domain wall energy density $U$, the electroelastic energy density $W$ and the last two terms in Eq. (5) remain unchanged by this transformation. The only modified energy term is the phase-separation potential $\chi$ which is converted to

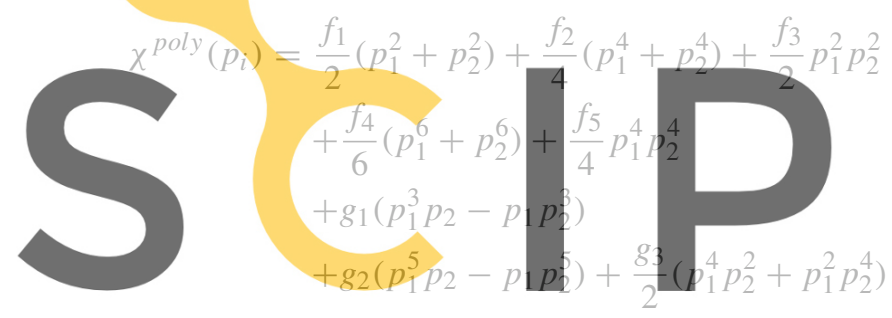

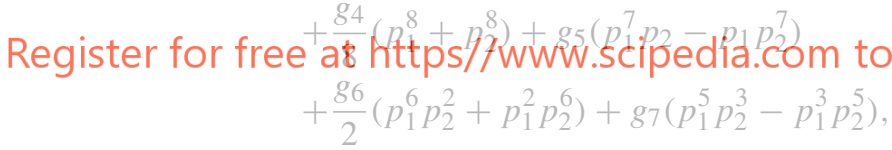

where

$$
\begin{aligned}
& f_{1}=a_{1}, \quad f_{2}=\frac{1}{4}\left[(3+\cos 4 \theta) a_{2}+(1-\cos 4 \theta) a_{3}\right] \\
& f_{3}=\frac{1}{4}\left[3(1-\cos 4 \theta) a_{2}+(1+3 \cos 4 \theta) a_{3}\right] \\
& f_{4}=\frac{1}{8}(5+3 \cos 4 \theta) a_{4} \\
& f_{5}=\frac{1}{64}(9+20 \cos 4 \theta+35 \cos 8 \theta) a_{5} \\
& g_{1}=\frac{1}{4}\left(a_{2}-a_{3}\right) \sin 4 \theta, \\
& g_{2}=\frac{1}{4} a_{4} \sin 4 \theta, \quad g_{3}=\frac{5}{8} a_{4}(1-\cos 4 \theta) \\
& g_{4}=\frac{1}{64} a_{5}(3-4 \cos 4 \theta+\cos 8 \theta) \\
& g_{5}=\frac{1}{64} a_{5}(-2 \sin 4 \theta+\sin 8 \theta)
\end{aligned}
$$

$g_{6}=\frac{1}{64} a_{5}(3+4 \cos 4 \theta-7 \cos 8 \theta)$,

$g_{7}=\frac{1}{64} a_{5}(-2 \sin 4 \theta-7 \sin 8 \theta)$.

By replacing $\chi$ with $\chi^{\text {poly }}$ in Eq. (5), the electromechanical enthalpy density for a ferroelectric polycrystal takes the form

$$
\begin{aligned}
h^{\text {poly }}(\boldsymbol{\varepsilon}, \mathbf{p}, \nabla \mathbf{p}, \mathbf{E}, v)= & \left(v^{2}+\eta_{\kappa}\right)[U(\nabla \mathbf{p})+W(\mathbf{p}, \boldsymbol{\varepsilon})] \\
& +\chi^{\text {poly }}(\mathbf{p})-\frac{\varepsilon_{0}}{2}|\mathbf{E}|^{2}-\mathbf{E} \cdot \mathbf{p} .
\end{aligned}
$$

Finally, the total electromechanical enthalpy in Eq. (4) is formulated for a possibly fractured ferroelectric polycrystal by considering Eas. (12) and (16) as
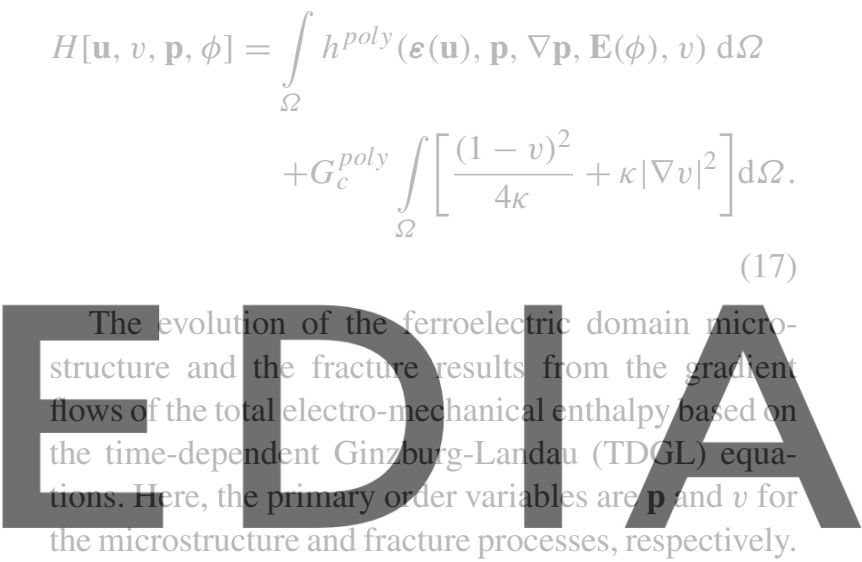

Thus, the governing equations, are obtained by assumdomn hathe the versign whithout the watermark

immediately to mechanical and electrostatic equilibrium (with infinite mobility), i.e.

$$
\begin{aligned}
& \mu_{p} \int_{\Omega} \dot{p}_{i} \delta p_{i} \mathrm{~d} \Omega=-\int_{\Omega} \frac{\partial h^{\text {poly }}}{\partial p_{i}} \delta p_{i} \mathrm{~d} \Omega, \\
& \mu_{v} \int_{\Omega} \dot{v} \delta v \mathrm{~d} \Omega=-\int_{\Omega} \frac{\partial h^{\text {poly }}}{\partial v} \delta v \mathrm{~d} \Omega, \\
& 0=\int_{\Omega} \sigma_{i j} \delta \varepsilon_{i j} \mathrm{~d} \Omega, \\
& 0=\int_{\Omega} D_{i} \delta E_{i} \mathrm{~d} \Omega,
\end{aligned}
$$

where $1 / \mu_{p}>0$ and $1 / \mu_{v}>0$ are the mobilities of the processes. The stresses and electric displacements are derived from the enthalpy density, respectively, as $\boldsymbol{\sigma}=\partial h^{\text {poly }} / \partial \boldsymbol{\varepsilon}$ and $\mathbf{D}=-\partial h^{\text {poly }} / \partial \mathbf{E}$.

The weak forms of the evolution and equilibrium equations are discretized in space with standard finite 
elements. Equations (18) and (19) are discretized in time with a semi-implicit scheme. A simple algorithm to solve the coupled system in a straightforward staggered approach is presented next. This algorithm describes how to advance in one load step $w^{n}$, and it is meant to achieve steady states for both ferroelectric domains and fracture processes in each load step. The functions $\mathbf{g}\left(w^{n}\right)$ and $f\left(w^{n}\right)$ encode the Dirichlet data for the mechanical displacement and electric potential as a function of the load step. After reaching a steady state for both the polarization $\mathbf{p}$ and the phase-field $v$, the values for $v^{n}, \mathbf{u}^{n}, \mathbf{p}^{n}$ and $\phi^{n}$ are recorded and the algorithm proceeds to a new load step $w^{n+1}$.

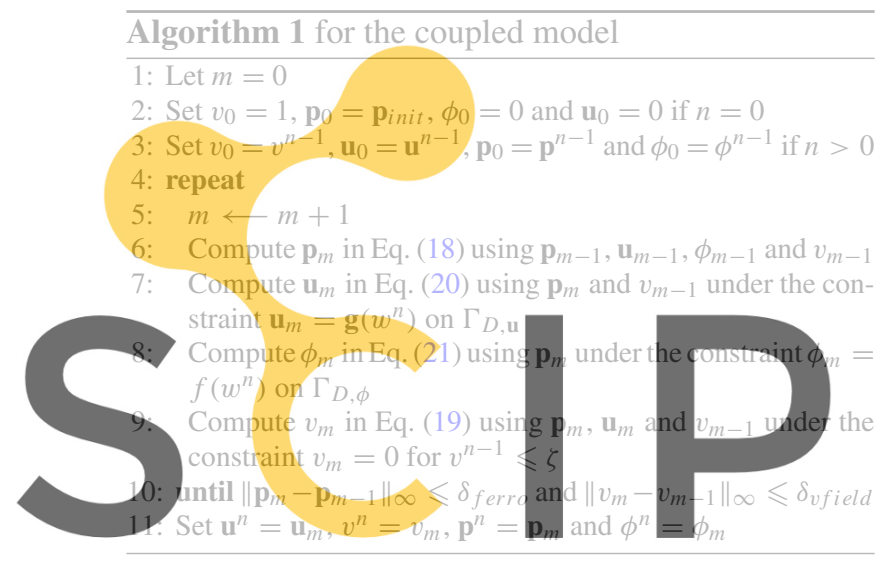

Register for free at https//www.scipedia.com to

\section{Numerical simulations and discussion}

We consider a rectangular domain of normalized dimensions $200 \times 200$ according to the normalizations presented in Table 1 . It is discretized with approximately 80,000 triangular finite elements of size $h \simeq 1$. First, the phase-field model of grain growth described in Sect. 2.1 is used to obtain different polycrystalline microstructures. The parameters of the kinetic equation (3) are selected as $\alpha=1, \beta=1, \gamma=1$, $\kappa_{i}=0.5$ and $\mu_{g}=1$. The time step $\Delta t=0.25$ is also chosen for the numerical integration scheme. Twenty field variables $(m=20)$ are considered and they are initialized with random small variables as $-0.001<\eta_{i}(i=1, \ldots, 20)<0.001$. Periodic boundary conditions are applied. The simulation is carried out on parallel processors for 7,000 steps using the finite element library of the Kratos multi-physics package
Table 1 Normalization of the variables and parameters $(i=$ $1,2,3)$

\begin{tabular}{lllll}
\hline$x_{i}^{\prime}$ & $p_{i}^{\prime}$ & $t^{\prime}$ & $\varepsilon_{0}^{\prime}$ & \\
\hline$x_{i} \sqrt{c_{0} / a_{0}} / p_{0}$ & $p_{i} / p_{0}$ & $t c_{0} / \mu p_{0}^{2}$ & $\varepsilon_{0} c_{0} / p_{0}^{2}$ & \\
\hline$\phi^{\prime}$ & $a_{1}^{\prime}$ & $a_{2}^{\prime}$ & $a_{3}^{\prime}$ & $a_{4}^{\prime}$ \\
\hline$\phi / \sqrt{a_{0} c_{0}}$ & $a_{1} p_{0}^{2} / c_{0}$ & $a_{2} p_{0}^{4} / c_{0}$ & $a_{3} p_{0}^{4} / c_{0}$ & $a_{4} p_{0}^{6} / c_{0}$ \\
\hline$a_{5}^{\prime}$ & $b_{i}^{\prime}$ & $c_{i}^{\prime}$ & $G_{c}^{\prime}$ & \\
\hline$a_{5} p_{0}^{8} / c_{0}$ & $b_{i} p_{0}^{2} / c_{0}$ & $c_{i} / c_{0}$ & $G_{c} \sqrt{1 / a_{0} c_{0}} / p_{0}$ & \\
\hline
\end{tabular}

(Dadvand et al. 2010). The contour of $\xi$ in Eq. (11) is presented in Fig. 1 for two snapshots of the polycrystalline microstructure evolution. Grain boundaries are highlighted by darker regions in this figure. In the early stages of the simulation, the field variables $\eta_{i}$ start to grow at different locations of the domain towards the crystallization. After about 500 steps, a well defined microstructure can be observed in Fig. 1a which con-

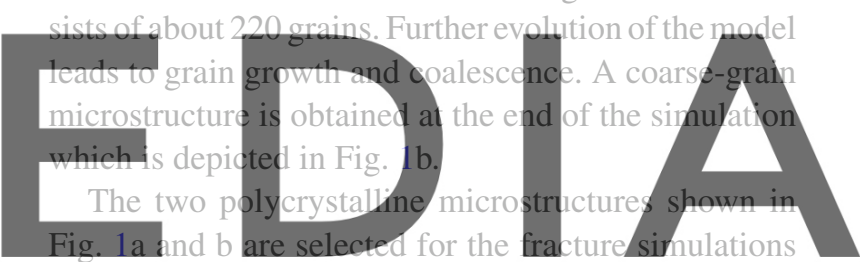

in a fine- and a coarse-grain ferroelectric polycrystal,

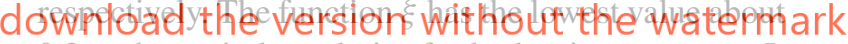
0.2 at the grain boundaries for both micostructures. It has been shown that if this ratio is below 0.3 , the fracture mode is entirely intergranular over a fine-grain microstructure (Sukumar et al. 2003). The grain orientations $\Theta_{i}(i=1, \ldots, 20)$ are assigned randomly between $0^{\circ}$ and $45^{\circ}$ using the function $\theta$ in Eq. (13).

For the fracture simulations, a monotonically increasing mechanical load is applied by pulling the top and bottom sides of the model with a uniform vertical mechanical displacement such that $\mathbf{u}_{ \pm}=(0, \pm w)$, where + and - indicate the top and bottom sides of the model respectively and $w$ is the load step, see Fig. 2. The vertical mechanical displacement is also constrained at the top half and bottom half of the left side such that $u_{2 \pm}= \pm w$. As this model does not have any pre-crack, this extra boundary condition is needed to force the crack to initiate at the center-left of the model. For all the simulations, the initial polarization $\mathbf{p}_{\text {init }}=(1,0)$ is assigned along the positive $x_{1}$ direction in the global coordinate system, see Fig. 2a. Note 


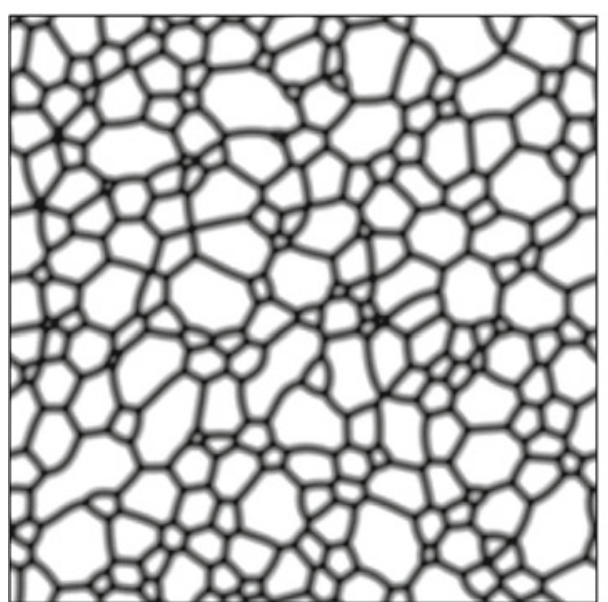

(a)

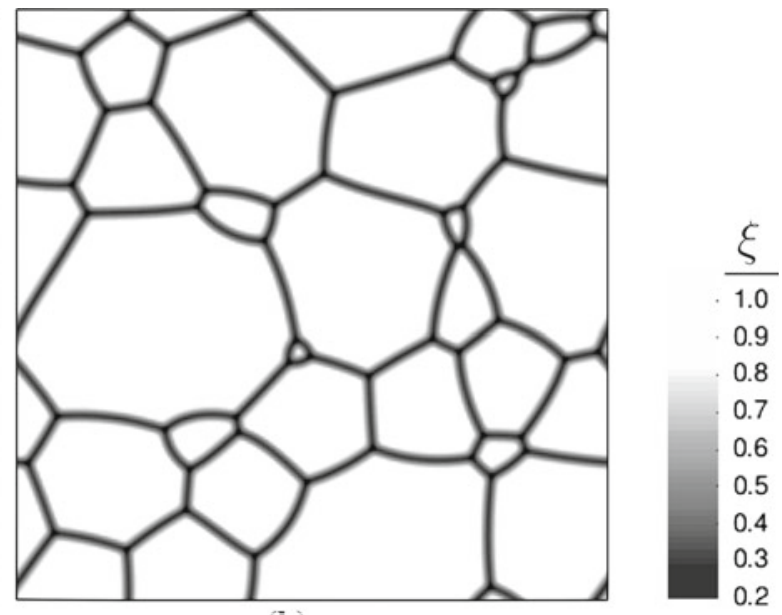

(b)

Fig. 1 Two snapshots of the polycrystalline microstructure evolution at time steps $\mathbf{a} t=125 \mathbf{b} t=1,750$. The gray contour indicates the distribution of $\xi$. Grain boundaries are highlighted by darker regions
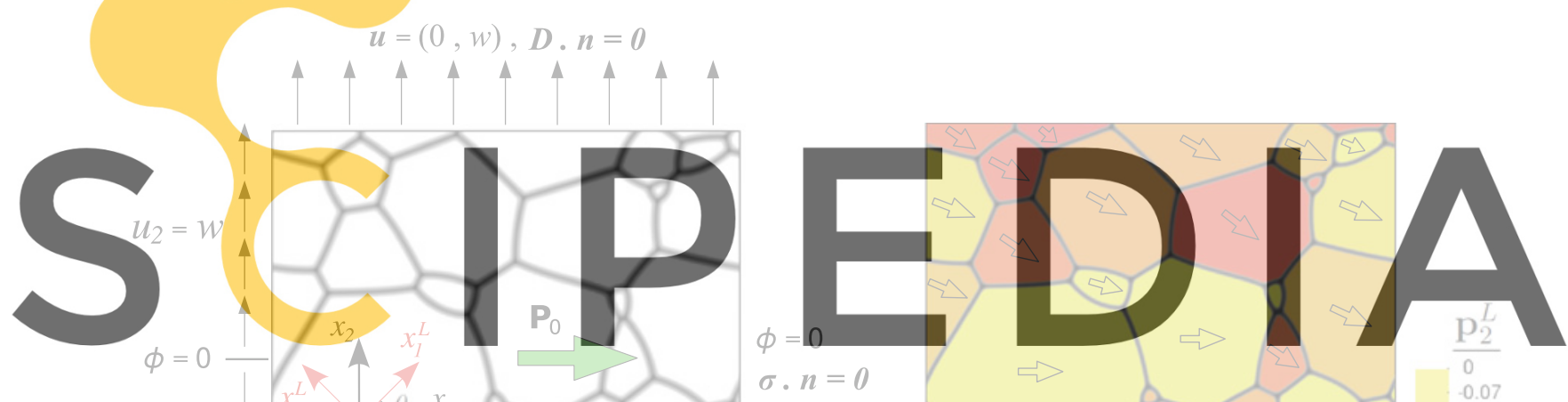

Register for free at httpst/www.scipedia.com

download the version without the watermark
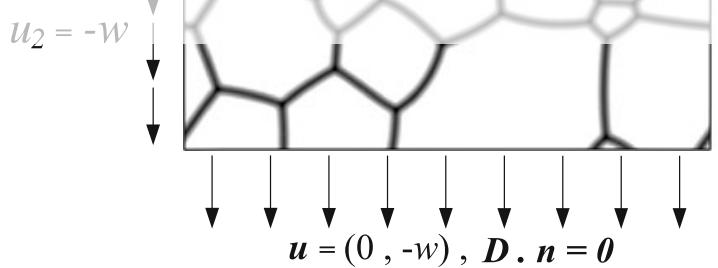

(a)

Fig. 2 a Computational model with boundary conditions; the initial polarization $\mathbf{p}_{0}$ is along the positive $x_{1}$ direction in the global coordinate system. The orientation of each grain is assigned randomly as $0^{\circ} \leqslant \theta \leqslant 45^{\circ}$ which indicates the angle between the global coordinate system $\left(x_{1}-x_{2}\right)$ of the polycrystal

that with the horizontal initial polarization and choosing the grain orientations between $0^{\circ}$ and $45^{\circ}$, ferroelastic switching becomes favorable with an acceptable mismatch between the grains, consequently leading to

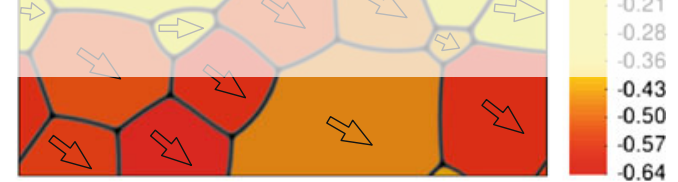

(b)

and the local coordinate system $\left(x_{1}^{L}-x_{2}^{L}\right)$ of each grain. Same boundary conditions are also applied for the fracture simulation of the fine-grain structure. b Distribution of the initial polarization in each grain. The orientations of the initial polarization vectors are indicated with arrows

strong interactions between the microstructure and the crack propagation. For clarification purposes, the initial polarization in each grain is presented in Fig. $2 b$ for the coarse-grain structure. As for the electrical boundary 
conditions, the electric potential on the left and right sides of the domain is set to 0 . It is also assumed that the normal component of the electric displacement vanishes on other surfaces, i.e. $\mathbf{D} \cdot \mathbf{n}=0$, representing the free-space. All the boundaries satisfy the free-polarization boundary condition.

For convenience, dimensionless variables are selected through the normalizations presented in Table 1. The constants are chosen to fit the behavior of single crystals of barium titanate $\left(\mathrm{BaTiO}_{3}\right)$ at room temperature, taking $c_{0}=1 \mathrm{GPa}$, a value for the spontaneous polarization of $p_{0}=0.26 \mathrm{C} / \mathrm{m}^{2}$, the relative spontaneous strains $\varepsilon_{a}=-0.44 \%$ along a-axis and $\varepsilon_{c}=0.65 \%$ along c-axis (Zhang and Bhattacharya 2005a; Wang et al. 2007). The domain wall scaling parameter is set to $a_{0}=3.7 \times 10^{-9} \frac{\mathrm{Vm}^{3}}{\mathrm{C}}$. The normalized scaling parameter of the domain wall energy $a_{0}^{\prime}$ is used to adjust the domain wall width in the computational domain. This parameter has to be chosen such that the variation of the polarization can be resolved by the discretization while domain walls remain sufficiently sharp relative

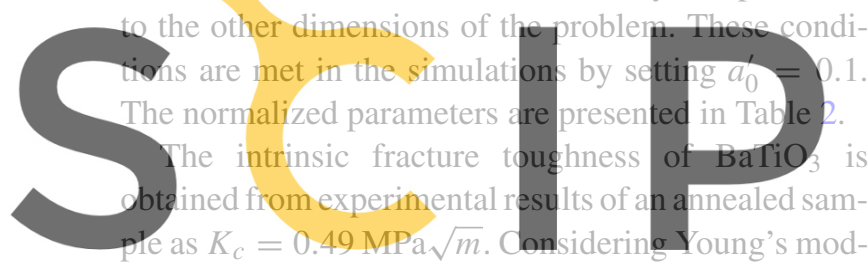

ulus and Poisson's ratio for $\mathrm{BaTiO}_{3}$ as $E=100 \mathrm{GPa}$

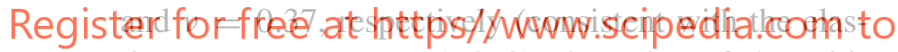
tic constants $\left.c_{i}, i=1,2,3\right)$, the value of the critical energy release rate in plane-strain is obtained as $G_{c}=\left(1-v^{2}\right) K_{c}^{2} / E=2 \mathrm{~J} / \mathrm{m}^{2}$. The value of normalized critical energy release rate is then calculated as $G_{c}^{\prime}$ $=4$. Using this value and the obtained distributions of $\xi$ in Fig. 1a and b, the critical energy release rate is defined over both microstructures in Eq. (12).

The residual stiffness $\eta_{\kappa}$ must be chosen as small as possible to avoid adding too much artificial stiffness and permittivity to the elements in the fracture zone, while preserving the non-singularity of the stiff-

Table 2 Normalized parameters

\begin{tabular}{lllllll}
\hline$a_{1}^{\prime}$ & $a_{2}^{\prime}$ & $a_{3}^{\prime}$ & $a_{4}^{\prime}$ & $a_{5}^{\prime}$ & $\varepsilon_{0}^{\prime}$ & $G_{c}^{\prime}$ \\
\hline-0.007 & -0.009 & 0.018 & 0.0261 & 5 & 0.131 & 4 \\
\hline$c_{1}^{\prime}$ & $c_{2}^{\prime}$ & $c_{3}^{\prime}$ & $b_{1}^{\prime}$ & $b_{2}^{\prime}$ & $b_{3}^{\prime}$ & $a_{0}^{\prime}$ \\
\hline 185 & 111 & 74 & 1.4282 & -0.185 & 0.8066 & 0.1 \\
\hline
\end{tabular}

ness matrices used for the solution of finite element equations. We take $\eta_{\kappa}=10^{-6}$ without any observed numerical instabilities in the simulations.

The value of the fracture regularization parameter $\kappa$ is chosen based on parametric studies of the discretized surface energy (Bourdin et al. 2008; Amor et al. 2009). These numerical experiments indicate that setting $\kappa \sim$ $h$ gives reasonable results, although the computed surface energy can be expected to be slightly overestimated. For the simulations of this paper, the regularization parameter is set to twice the element size as $\kappa=$ 2 , i.e. $h / \kappa=0.5$. The remaining constants are chosen as follows: two tolerances $\delta_{\text {ferro }}=10^{-3}$ and $\delta_{v \text { field }}$ $=2 \times 10^{-3}$, the threshold to detect the fracture zone $\zeta=2 \times 10^{-2}$ and the inverse of the mobilities $\mu_{p}=1$ and $\mu_{v}=15$. One hundred load steps are computed ( $n=100)$ with load increments of $\Delta w^{n}=3 \times 10^{-2}$. The normalized time step $\Delta t_{m}^{\prime}=10^{-2}$ leads to convergent and accurate solutions for the numerical time integration of the gradient flow equations in Eqs. (18) and (19).

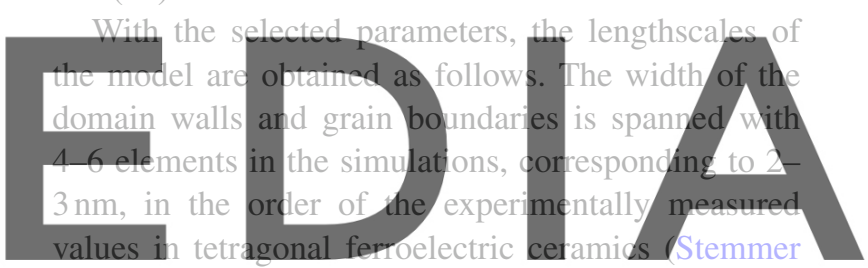

et al. 1995; Floquet et al. 1997; Tan and Shang 2002).

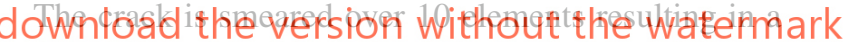
width of $5 \mathrm{~nm}$. The average grain sizes of the fine- and coarse-grain structures are obtained as 5 and $20 \mathrm{~nm}$, respectively. Note that the physical grain size of the ferroelectric polycrystals is in the order of micrometers. In this micrometer scale, extremely fine meshes would be needed to model the nanoscale features such as the domain walls and grain boundaries, demanding massive parallel processing. However, the present configuration provides a proof of concept of the model and allows for a qualitative study. More physically realistic computations are on the way.

Figures 3 and 4 present the final computed crack paths (when the sample is splitted into two parts) in the fine- and coarse-grain structures, respectively. It is obvious in Fig. 3 that the crack propagates mainly along the grain boundaries, demonstrating the intergranular mode of fracture in the fine-grain structure. On the other hand, transgranular crack propagation is observed in Fig. 4 where the crack propagates through the grains. Experimental observations in ferroelectric 


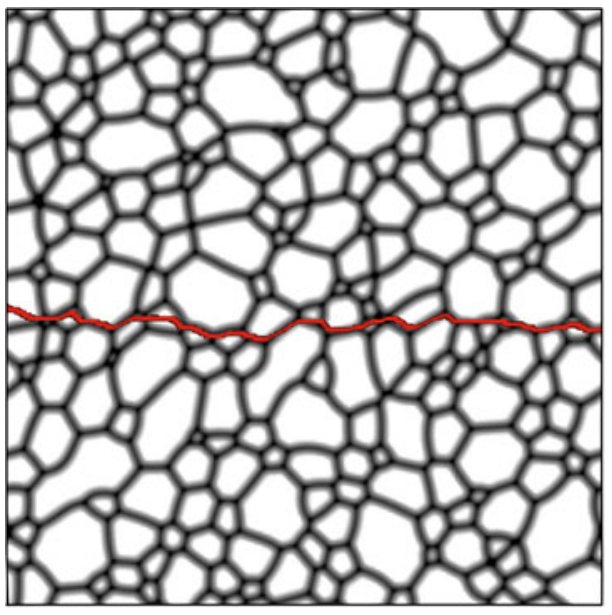

(a)

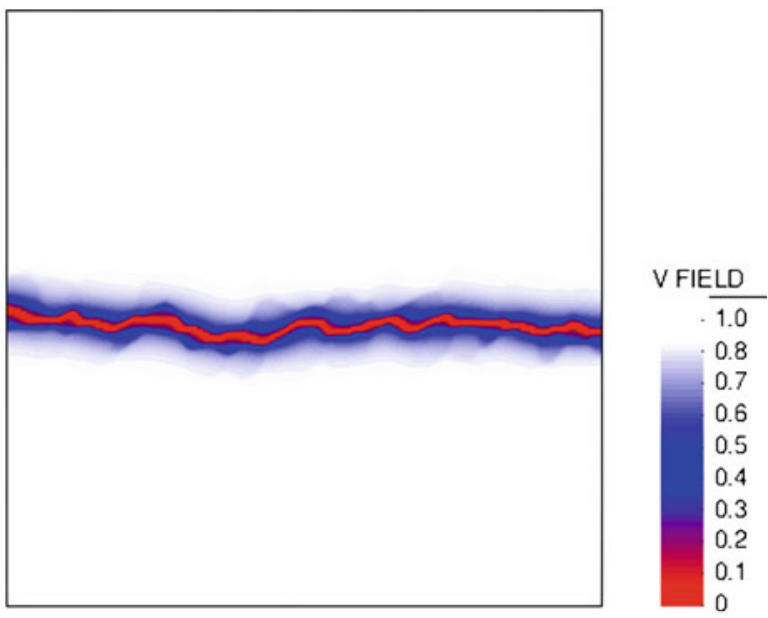

(b)

Fig. 3 Crack path in the fine-grain ferroelectric polycrystal a the points where $v=0$ are depicted in red to indicate the crack position b field $v$ representing the smeared crack
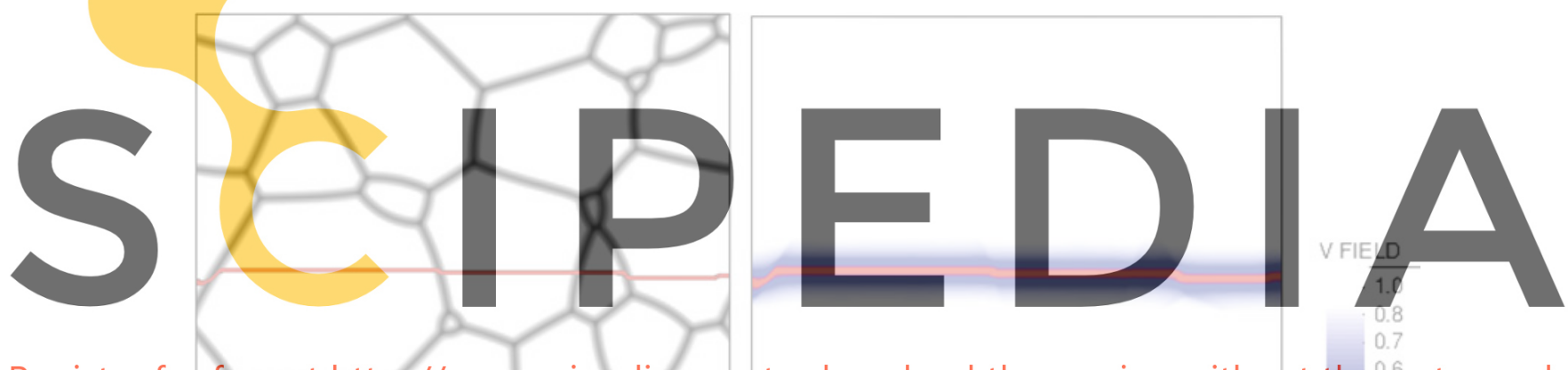

Register for free at https//www.scipedia.com to

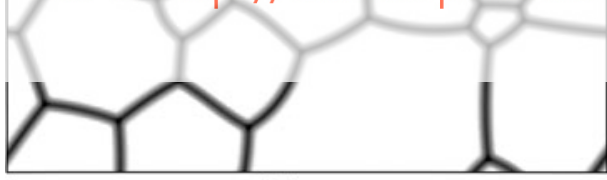

(a)

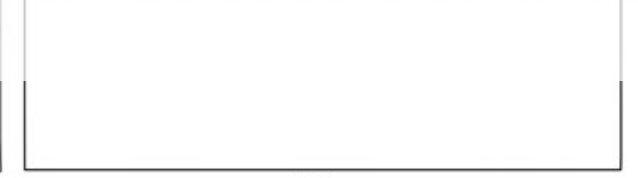

(b)

Fig. 4 Crack path in the coarse-grain ferroelectric polycrystal a the points where $v=0$ are depicted in red to indicate the crack position b field $v$ representing the smeared crack

polycrystals also show this transition from intergranular fracture for small grains to transgranular fracture in the case of large grains (Kim et al. 1990; Meschke et al. 1997).

To evaluate the effect of the fracture toughening mechanisms, the evolution of the normalized crack length (the second integral in Eq. (17) without the factor $G_{c}^{\text {poly }}$ ) is plotted in Fig. 5 as a function of the load step $w$. Both the fine- and coarse-grain graphs start from zero (intact model), and are almost iden- tical until the cracks initiate at load step $w=1.17$. After this point, the crack in the fine-grain structure starts to propagate abruptly, i.e. a small increase of the load leads to a big jump in the crack length. This is because after cutting the first grain, the crack starts to propagate faster along the grain boundaries with the lower fracture toughness. Interestingly, the abrupt propagation stops at load step $w=1.2$ (point $\mathbf{a}$ in the fine-grain graph). This crack arrest can be easily understood by tracking the position of the crack tip in 


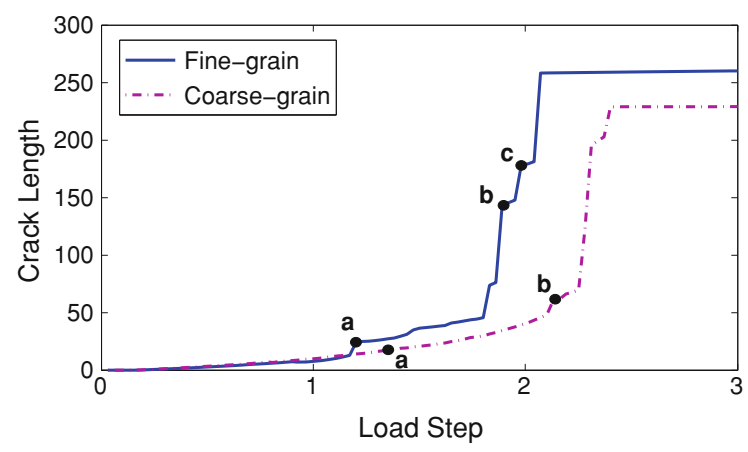

Fig. 5 Evolution of the normalized crack length as a function of the load step. The letters, marked in the graphs of the fineand coarse-grain structures, correspond to the load steps shown in Figs. 6 and 7, respectively

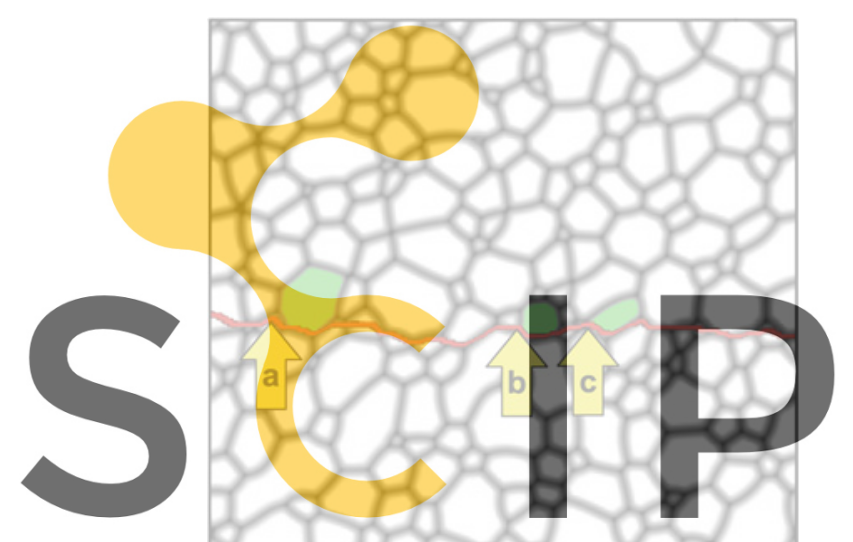

Register for free at https//www.scipedia.com to

Fig. 6 Three steps of the crack propagation in the fine-grain structure. The letters $a-c$ correspond to the load steps marked in the graph of the fine-grain structure in Fig. 5. In each load step, the position of the crack tip is indicated by the yellow arrow and the grain in front of the crack tip is highlighted with green. Crack deflection around the highlighted grains is obvious in this figure

this load step, as presented in Fig. 6. As the crack meets the first highlighted grain at load step $\mathbf{a}$, it is forced to move around the grain since the fracture toughness of the grain interior is higher then the grain boundary, leading to crack deflection along the grain boundary. This phenomena is the so-called crack deflection toughening in intergranular mode of fracture (Faber and Evans 1983; Kueck et al. 2008). The crack shows this behavior in other load steps $\mathbf{b}$ and $\mathbf{c}$, where the crack is arrested by the corresponding highlighted grains in Fig. 6.

Regarding the crack propagation in the coarsegrain microstructure, its crack-length graph in Fig. 5 shows a slower growth rate than the fine-grain structure. This is mainly due to the fact that, the transgranular mode of fracture in the coarse-grain structure allows the crack to interact with the ferroelectric domain microstructure inside the grains. Due to the high tensile stresses around the fracture zone, the polarization vectors start to rotate towards the vertical direction which leads to $90^{\circ}$ ferroelastic domain switching. Figure 7 presents two snapshots of the evolution of the ferroelectric domain microstructure. The formation of a multiple-domain structure ahead of the crack tip is evident in Fig. 7a. The switched regions form twins ahead of the crack tip. Since the crystal's unit cell is longer along the polarization direction, as encoded in the Landau-Devonshire model, compressive stresses in the vertical $\left(x_{2}\right)$ direction are induced by the vertical twins, leading to a strong toughening effect, already pointed out in the literature (Schneider 2007; Abdollahi and Arias 2011b). $90^{\circ}$ ferroelastic domain switching is also favorable in the grains with a high misalingment since the polarization vec-

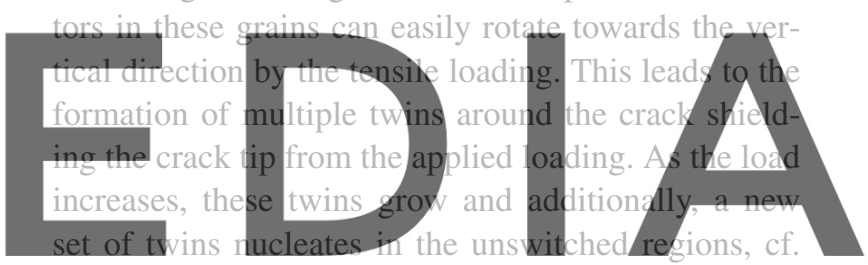

Fig. 7a and b. The slow crack propagation periods,

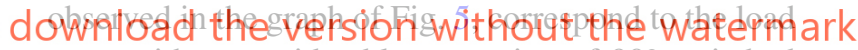
steps with a considerable expansion of $90^{\circ}$ switched domains. Therefore, the main toughening mechanism in a coarse-grain ferroelectric polycrystal is the domain switching-induced toughening. This toughening mechanism is also reported in experiments of crack propagation in $\mathrm{BaTiO}_{3}$ (Meschke et al. 1997, 2000; Fang et al. 2007). In contrast, $90^{\circ}$ ferroelastic switching is less pronounced in the fine-grain structure since the crack propagates faster along the grain boundaries with a lower applied load. It is obvious in Fig. 5 that the crack length in the fine-grain structure reaches its maximum value sooner than the coarse-grain structure. This maximum level correspond to the point when the crack splits the model into two parts. Therefore the effective fracture toughness of the coarse-grain structure is higher than the fine-grain, which agrees with experiments (Meschke et al. 1997). Due to the crack deflection in the fine-grain structure, the final length of the crack is larger than that of the coarse-grain structure. 


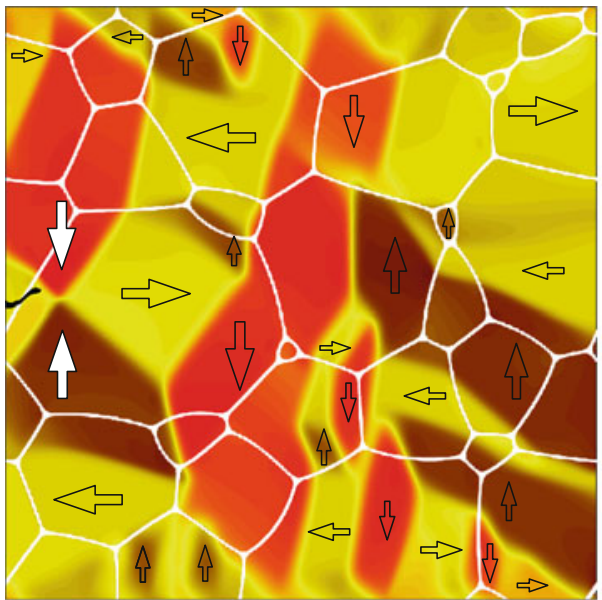

(a)

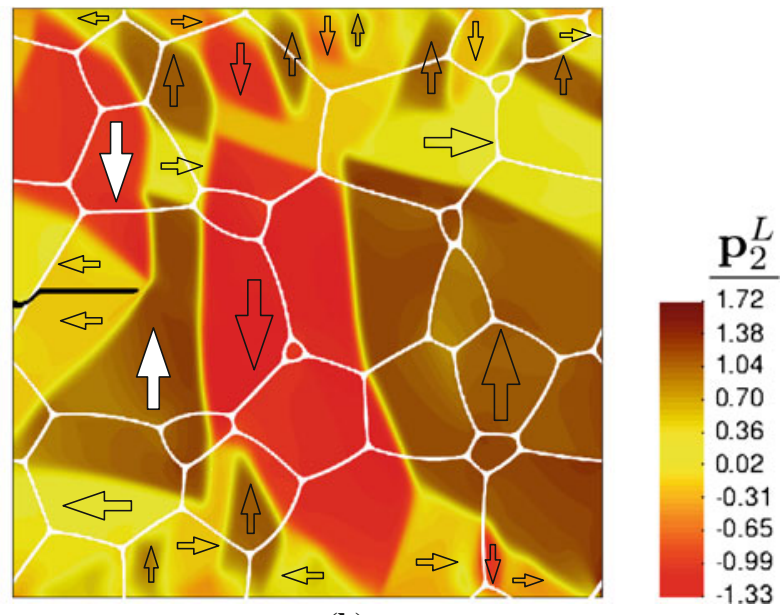

(b)
Fig. 7 Two snapshots of the evolution of the ferroelectric domain microstructure and the crack path at load steps a $w=1.32, \mathbf{b} w$ $=2.1$. These figures correspond to the load steps marked in the crack length graph of the coarse-grain structure in Fig. 5. The local vertical polarization $\mathbf{p}_{2}^{L}$ highlights the domain structure.
Domain orientations are indicated with arrows, which are bold white for the twins ahead of the crack. The points where $v=0$ are represented in black to show the crack position. Grain boundaries are highlighted by white lines

\section{Conclusions}

We perform, to the best of our knowledge, the first simulation of crack propagation in ferroelectric polycrystals with the goal of qualitatively evaluating the effect of the grain size and ferroelectric domain microstructures on the fracture process. For this purpose, we extend to polycrystals a phase-field model of fracture in ferroelectric single-crystals by incorporating the differential fracture toughness of the bulk and grain boundaries, and different crystal orientations of the grains. Simulation results show intergranular and transgranular crack propagations in fine- and coarse-grain structures, respectively. Crack deflection is observed as the main toughening mechanism in the intergranular mode of fracture in the fine-grain structure. On the other hand, as transgranular fracture is the dominant mode in coarse-grain microstructures, the crack interacts with the ferroelectric domains inside the grains. In particular, $90^{\circ}$ ferroelastic domain switching results in a considerable fracture toughening effect. All these observations agree with experiment.

We also suggest that more work is needed for a quantitative evaluation of the combined effect of the polycrystalline microstructure and the ferroelectric domain microstructure on the fracture response of ferroelectric polycrystals. In particular, the relative physical size of the grains and ferroelectric domains should be considered for more realistic simulations. Besides the grain size, the model presents three lengthscales: (1) the width of the grain boundaries, (2) the width of the ferroelectric domain walls, and (3) the width of the smeared cracks. The first two lengthscales can be estimated experimentally (Stemmer et al. 1995; Floquet et al. 1997; Tan and Shang 2002). The latter is a numerical artifact, which could have a significant effect on the simulation results. A sensitivity analysis on the regularization parameter of the fracture phase-field relative to the other lengthscales of the problem would be necessary, as well as an extension to three dimensions. Although domain switching is claimed to be the main toughnening mechanism in coarse-grain ferroelectric polycrystals, the intrinsic anisotropy of the crystal in each grain, i.e. the different surface energy in different cleavage planes, is bound to have an effect as well. Besides, there is evidence that the properties of the grain boundaries depend on the crystallographic misorientation of the neighboring grains. Another important issue is the quantification and relative magnitude of the mobility parameters $\mu_{p}$ and $\mu_{v}$, which can have an important effect on the resulting response. These topics are currently under investigation.

Finally, in the context of the multiscale paradigm, the model proposed here targets a mesoscale, 
half-way between atomistic models and phenomenological macro-scale models. Although, the bridging to the smaller and larger scale models is beyond the scope of the present paper, one can readily envision some connections. On one side, one can extract valuable information about the parameters of the phase-field model from atomistic calculations, possibly ab initio. Parameters susceptible of atomistic feed are those defining the Ginzburg-Laundau model, the mobility of the domain walls, the fracture toughness along cleavage planes and the structure of the grain boundaries, to name a few. On the other side, our ability to model polycrystalline structures and eventually three dimensional systems will allow us to simulate the response of representative volume elements in a micromechanical approach to extract effective properties for macroscopic phenomenological models.

Acknowledgments The authors gratefully acknowledge the support of the Ministerio de Ciencia e Innovación (DPI201019145).

\section{References}

Abdollahi A, Arias I (2011a) Modeling and numerical simulation of crack propagation in piezoelectric and ferroelectric materials considering different crack face conditions (submitted for publication)

Abdollahi A, Arias I (2011b) Phase-field modeling of the coupled microstructure and fracture evolution in ferroelectric single crystals. Acta Mater 59:4733-4746

Abdollahi A, Arias I (2011c) Phase-field simulation of anisotropic crack propagation in ferroelectric single crystals: effect of microstructure on the fracture process. Model Simul Mater Sci Eng 19:074010

Amor H, Marigo JJ, Maurini C (2009) Regularized formulation of the variational brittle fracture with unilateral contact: numerical experiments. J Mech Phys Solids 57:1209-1229

Bourdin B, Francfort GA, Marigo JJ (2008) The variational approach to fracture. J Elast 91:5-148

Dadvand P, Rossi R, Onate E (2010) An object-oriented environment for developing finite element codes for multi-disciplinary applications. Arch Comput Methods Eng 17:253297

DeSimone A, Kohn RV, Müller S, Otto F, Schäfer R (2001) Twodimensional modeling of soft ferromagnetic films. Proc R Soc Lond A 457:2983-2991

Devonshire AF (1949) Theory of barium titanate. 1. Philos Mag 40:1040-1063

Devonshire AF (1951) Theory of barium titanate. 2. Philos Mag 42:1065-1079

Faber KT, Evans AG (1983) Intergranular crack-deflection toughening in silicon-carbide. J Am Ceram Soc 66:C94C96
Fan D, Chen LQ (1997) Computer simulation of grain growth using a continuum field model. Acta Mater 45:611-622

Fang DN, Jiang YJ, Li S, Sun CT (2007) Interactions between domain switching and crack propagation in poled $\mathrm{BaTiO} 3$ single crystal under mechanical loading. Acta Mater 55:5758-5767

Floquet N, Valot CM, Mesnier MT, Niepce JC, Normand L, Thorel A, Kilaas R (1997) Ferroelectric domain walls in BaTiO3: fingerprints in XRPD diagrams and quantitative HRTEM image analysis. J Phys III 7:1105-1128

Francfort GA, Marigo JJ (1998) Revisiting brittle fracture as an energy minimization problem. J Mech Phys Solids 46:1319-1342

Grah M, Alzebdeh K, Sheng PY, Vaudin MD, Bowman KJ, Ostoja-Starzewski M (1996) Brittle-intergranular failure in 2D microstructures: experiments and computer simulations. Acta Mater 44:4003-4018

Griffith AA (1921) The phenomena of rupture and flow in solids. Philos Trans Royal Soc Lond A 221:163-198

Hackemann S, Pfeiffer W (2003) Domain switching in process zones of PZT: characterization by microdiffraction and fracture mechanical methods. J Eur Ceram Soc 23:141-151

Huber JE (2005) Micromechanical modelling of ferroelectrics. Curr Opin Solid State Mater Sci 9:100-106

Jones JL, Motahari SM, VarlioglU M, Lienert U, Bernier JV, Hoffman M, Uestuendag E (2007) Crack tip process zone domain switching in a soft lead zirconate titanate ceramic. Acta Mater 55:5538-5548

Kamlah M (2001) Ferroelectric and ferroelastic piezoceramics-modeling of electromechanical hysteresis phenomena. Cont Mech Therm 13:219-268

Kim SB, Kim DY, Kim JJ, Cho SH (1990) Effect of grain-size and poling on the fracture mode of lead zirconate titanate ceramics. J Am Ceram Soc 73:161-163

Kueck AM, Kim DK, Ramasse QM, De Jonghe LC, Ritchie RO (2008) Atomic-resolution imaging of the nanoscale origin of toughness in rare-earth doped SiC. Nano Lett 8:29352939

Kuna M (2010) Fracture mechanics of piezoelectric materialswhere are we right now?. Eng Fract Mech 77:309-326

Landis CM (2003) On the fracture toughness of ferroelastic materials. J Mech Phys Solids 51(8):1347-1369

Li Q, Kuna M (2011) Evaluation of electromechanical fracture behavior by configurational forces in cracked ferroelectric polycrystals. Comput Mater Sci. doi:10.1016/j.commatsci. 2011.01.050

Li W, Landis CM (2011) Nucleation and growth of domains near crack tips in single crystal ferroelectrics. Eng Fract Mech. doi:10.1016/j.engfracmech.2011.01.002

Meschke F, Kolleck A, Schneider GA (1997) R-curve behaviour of $\mathrm{BaTiO}_{3}$ due to stress-induced ferroelastic domain switching. J Eur Ceram Soc 17:1143-1149

Meschke F, Raddatz O, Kolleck A, Schneider GA (2000) R-curve behavior and crack-closure stresses in barium titanate and (Mg, Y)-PSZ ceramics. J Am Ceram Soc 83:353361

Schneider GA (2007) Influence of electric field and mechanical stresses on the fracture of ferroelectrics. Annu Rev Mater Res 37:491-538 
Sheng JS, Landis CM (2007) Toughening due to domain switching in single crystal ferroelectric materials. Int $\mathrm{J}$ Fract 143:161-175

Shu YC, Bhattacharya K (2001) Domain patterns and macroscopic behaviour of ferroelectric materials. Phil Mag B 81:2021-2054

Song YC, Soh AK, Ni Y (2007) Phase field simulation of crack tip domain switching in ferroelectrics. J Phys D Appl Phys 40:1175-1182

Stemmer S, Streiffer SK, Ernst F, Ruhle M (1995) Atomistic structure of 90-degrees domain-walls in ferroelectric $\mathrm{PbTiO}_{3}$ thin-films. Phil Mag A 71:713-724

Sukumar N, Srolovitz DJ, Baker TJ, Prevost JH (2003) Brittle fracture in polycrystalline microstructures with the extended finite element method. Int J Numer Methods Eng 56:2015-2037

Tan XL, Shang JK (2002) In-situ transmission electron microscopy study of electric-field-induced grain-boundary cracking in lead zirconate titanate. Phil Mag A 82:1463-1478

Verhoosel CV, Gutierrez MA (2009) Modelling inter- and transgranular fracture in piezoelectric polycrystals. Eng Fract Mech 76:742-760

Wang J, Landis CM (2006) Effects of in-plane electric fields on the toughening behavior of ferroelectric ceramics. J Mech Mater Struct 1:1075-1095

Wang J, Zhang TY (2007) Phase field simulations of polarization switching-induced toughening in ferroelectric ceramics. Acta Mater 55:2465-2477

Wang J, Zhang TY (2008) Phase field simulations of a permeable crack parallel to the original polarization direction in a ferroelectric mono-domain. Eng Fract Mech 75(17): 48864897. doi:10.1016/j.engfracmech.2008.06.025

Wang YL, Tagantsev AK, Damjanovic D, Setter N, Yarmarkin VK, Sokolov AI, Lukyanchuk IA (2007) Landau thermodynamic potential for BaTiO3. J Appl Phys 101(1-9):104,115

Xu BX, Schrade D, Gross D, Mueller R (2010) Fracture simulation of ferroelectrics based on the phase field continuum and a damage variable. Int J Fract 166:163-172

Yang L, Dayal K (2011) Microstructure and stray electric fields at surface cracks in ferroelectrics. Int J Fract. doi:10.1007/ s10704-011-9670-2

Yang W, Zhu T (1998) Switch-toughening of ferroelectrics subjected to electric fields. J Mech Phys Solids 46:291-311

Zhang TY, Gao CF (2004) Fracture behaviors of piezoelectric materials. Theor Appl Fract Mech 41:339-379

Zhang W, Bhattacharya K (2005a) A computational model of ferroelectric domains. Part I: model formulation and domain switching. Acta Mater 53:185-198

Zhang W, Bhattacharya K (2005b) A computational model of ferroelectric domains. Part II: grain boundaries and defect pinning. Acta Mater 53:199-209

Zhu T, Yang W (1997) Toughness variation of ferroelectrics by polarization switch under non-uniform electric field. Acta Mater 45:4695-4702

Zhu T, Yang W (1999) Fatigue crack growth in ferroelectrics driven by cyclic electric loading. J Mech Phys Solids 47: 81-97 